\title{
Energy Efficient Channel Coding of Physiological Signals in Wireless Body Area Networks
}

\author{
Bafrin Zarei, Vallipuram Muthukkumarasamy and Xin-Wen Wu \\ Griffith University \\ Gold Coast, Australia
}

\begin{abstract}
An energy-efficient Forward Error Correction code (FEC), which is presented in this article, is designed specifically for wireless body area network (WBAN) with a focus towards pervasive healthcare applications. WBAN consists of wireless sensor nodes attached to the human body to monitor vital signs such as body temperature, activity or heart-rate. The network adopts a master-slave architecture, where the bodyworn slave node periodically sends sensor readings to a central master node. Similar to other wireless sensor network protocols, one of the primary design goals is to achieve low power consumption. Thus, for the wireless communication between sensor nodes on the body, no FEC is normally applied and redundancy is avoided. When error occurs, in noisy wireless channels, the entire message is retransmitted.
\end{abstract}

We designed a channel coding mechanism based on a Residue Number System (RNS) which can convert a binary weighted value to smaller independent residues. The residue to binary converter is the main energy consuming part of RNS. In this research, the reverse converter for a five-moduli set is presented based on the Mixed Radix Conversion method. This converter uses the formulated multiplicative inverse values in order to simplify the calculations and the required hardware for its implementation. The hardware requirements and conversion time of the proposed reverse convertor are calculated and compared with the other moduli set to illustrate its efficiency.

\section{General Terms}

Reliability, Channel Coding, Energy Efficiency, Body Area Networks.

\section{Keywords}

Residue Number Systems (RNS), Moduli Sets, Reverse Converters, Bit Error Rate (BER).

\section{INTRODUCTION}

A Wireless Body Area Network (WBAN) is a special type of Wireless Sensor Networks (WSNs). Advances in wireless communications enable a wide acceptance in WBAN, specifically in healthcare monitoring which is one of the most studied applications of WBAN. This new technology provides more effective management for healthcare delivery. In particular, monitoring of vital signs using wireless sensors is a fast growing area in modern healthcare due to its potential to arrest the unsustainable escalation of healthcare cost. WBAN facilitates continuous monitoring of increasing number of people with chronic conditions [1], [2].
Since WSN are deployed with battery-powered sensors, which are connected wirelessly, researchers try to design energy-efficient solutions to increase the lifetime of the nodes $[4,5]$. In [6, 7], The residue number system (RNS) is used to perform error correction in WSN with reasonable energy consumption. The RNS has already been used in applications such as FIR filtering [8,9]. It has been shown that improvements in the complexity of a reverse converter may lead to a significant decrease in the energy consumption.

The tiny battery-dependant wireless sensors are capable of sensing, processing and communicating with each other. The vital signs monitoring systems are typically area and power inefficient and usually depend on MAC protocols such as Bluetooth and 802.11 [3]-[6]. MAC protocols in all-purpose WSN have been focused on in many studies [6], [7], [9], [10]. Nevertheless, they are also not well suited to these specific biomedical WBAN applications. For instance, ZigBee/IEEE 802.15.4 [6] which is basically designed for small network devices with limited energy resources, lacks the cross-layer optimization features.

This paper describes a channel coding method based on RNS designed specifically for WBAN focusing on pervasive healthcare applications to tackle erroneous transmission. Similar to the other wireless sensor network MAC protocols, our primary design goal is to minimize the area and delay complexity of the applied code and thereby, minimize energy consumption. This is achieved through a focus on simplifying the reverse convertor which is the primary source for energy drain.

RNS is defined based on a moduli set, in which the residue to binary converters, known as reverse converter, has higher complexity compared to the binary to RNS converters known as the forward converter [1]. Therefore, the complexity of the reverse converter needs to be hardware-efficient and time-efficient. Moreover, the dynamic range of RNS is highly dependent on the selection of the moduli set.

The well-known three moduli set $\left\{2^{\mathrm{n}}-1,2^{\mathrm{n}}, 2^{\mathrm{n}}+1\right\}$ has been examined comprehensively in the literature $[2,3]$. One of the advantages of these moduli set is it has simple forward and reverse converters. Additionally, the modular arithmetic is more cost effective for these forms of moduli.

This paper considers the RNS to binary conversion problem of the moduli set $\left\{2^{n}+3,2^{n}+1,2^{n}, 2^{n}-1,2^{n}-3\right\}$. Therefore, it still keeps the set of the popular three-moduli set $\left\{2^{\mathrm{n}}-1,2^{\mathrm{n}}, 2^{\mathrm{n}}+1\right\}$ for high-speed and low cost residue 
to binary conversion. The reason for choosing this moduli set is to use it as a channel coder in WBAN applications. If we consider two of the moduli as redundant, the valid representation range is $3 n$ bits. Therefore, the network is enabled to recover up to $\mathrm{n}$ bit burst errors, which are limited to one of the receiver residues in the base station side. The other advantage of these moduli set is that each modulus can be represented by $\mathrm{n}$ or $\mathrm{n}+1$ bits. Therefore, it is a symmetric set and in the case of fixed framing, there will be almost no wastage of bits.

The residue number system components and the basic reverse converters are defined in Section 2. The proposed design of the RNS to binary convertor is presented in Section 3. Section 4 illustrates the area and conversion time trade off of the proposed converters in comparison with the existing fivemoduli set converter. Section 5 discusses the improvements of applying the proposed FEC, and finally Section 6 concludes the paper.

\section{BACKGROUND}

This section discusses the Residue Number System definitions and the reverse conversion fundamentals.

\subsection{Residue Number System}

Mutually prime integers $\mathrm{m}_{1}, \mathrm{~m}_{2}, \mathrm{~m}_{3}, \ldots, \mathrm{m}_{\mathrm{n}}$, called moduli in an RNS, determine the dynamic range or operating range as the product of the moduli, Mop $=\prod_{\mathrm{i}=1}^{\mathrm{n}} \mathrm{m}_{\mathrm{i}}$. The numbers between 0 and Mop -1 are represented by a unique set of residues. Thus, this system can represent a large number by using several smaller numbers. On the other hand, the results of the residual computation need to be combined to form the original number using an RNS to binary converter. The binary number $X$ can be shown as the residues $\left(\mathrm{x}_{1}, \mathrm{x}_{2}, \mathrm{x}_{3}, \ldots, \mathrm{x}_{\mathrm{n}}\right)$ in the RNS $\left[\mathrm{m}_{1}, \mathrm{~m}_{2}, \mathrm{~m}_{3}, \ldots, \mathrm{m}_{\mathrm{n}}\right]$, where $\mathrm{x}_{i}$ is the remainder of $X$ to $\mathrm{m}_{\mathrm{i}}$ or in other words $\mathrm{x}_{i}=|X|_{m_{i}}$.

\subsection{Reverse Conversion Concepts}

Two basic methods to convert number from RNS representation to its binary form are Chinese Remainder Theorem (CRT) and the Mixed Radix Conversion (MRC). The binary number $\mathrm{X}$ can be derived using CRT as $\mathrm{X}=$ $\left|\sum_{\mathrm{i}=1}^{\mathrm{n}} \mathrm{x}_{\mathrm{i}} \mathrm{M}_{\mathrm{i}}^{-1} \mathrm{M}_{\mathrm{i}}\right|_{\text {Mop }}$, where $\mathrm{M}_{\mathrm{i}}=\mathrm{M}_{\mathrm{op}} / \mathrm{m}_{\mathrm{i}}$, and the multiplicative inverse of $M_{i}$ is $M_{i}^{-1}$ where $\left|M_{i}^{-1} M_{i}\right|_{m_{i}}=1$.

In the MRC, the decoded number can be represented as

$X=d_{1}+d_{2} m_{1}+d_{3} m_{1} m_{2}+\cdots+d_{n} m_{1} m_{2} \ldots m_{n-1}$

where the mixed radix digit can be obtained as:

$$
\begin{aligned}
& \mathrm{d}_{1}=\left|\mathrm{x}_{1}\right|_{\mathrm{m}_{1}}=\mathrm{x}_{1} \\
& \mathrm{~d}_{2}=\left.\left.\left|\left(\mathrm{x}_{2}-\mathrm{x}_{1}\right)\right| \mathrm{m}_{1}^{-1}\right|_{\mathrm{m}_{2}}\right|_{\mathrm{m}_{2}} \\
& \mathrm{~d}_{3}=\left.\left.\left|\left(\left(\mathrm{x}_{3}-\mathrm{x}_{1}\right)\left|\mathrm{m}_{1}^{-1}\right|_{\mathrm{m}_{3}}-\mathrm{d}_{2}\right)\right| \mathrm{m}_{2}^{-1}\right|_{\mathrm{m}_{3}}\right|_{\mathrm{m}_{3}}
\end{aligned}
$$

and in the general case as;

$$
\begin{aligned}
& \mathrm{d}_{\mathrm{n}}= \\
& \left.\left|\left(\left(\mathrm{x}_{\mathrm{n}}-\mathrm{d}_{1}\right)\left|\mathrm{M}_{1}^{-1}\right|_{\mathrm{m}_{\mathrm{n}}}-\mathrm{d}_{2}\right)\right| \mathrm{M}_{2}^{-1}\right|_{\mathrm{m}_{\mathrm{n}}} \ldots-\left.\mathrm{d}_{\mathrm{n}-1}\left|\mathrm{M}_{\mathrm{n}-1}^{-1}\right|_{\mathrm{m}_{\mathrm{n}}}\right|_{\mathrm{m}_{\mathrm{n}}}
\end{aligned}
$$

\section{RESIDUE TO BINARY CONVERTER}

Consider the 5-moduli set $\left\{m_{1}, m_{2}, m_{3}, m_{4}, m_{5}\right\}=\left\{2^{\mathrm{n}}+\right.$ $\left.3,2^{\mathrm{n}}+1,2^{\mathrm{n}}, 2^{\mathrm{n}}-1,2^{\mathrm{n}}-3\right\}$ with five corresponding residues $\left\{\mathrm{x}_{1}, \mathrm{x}_{2}, \mathrm{x}_{3}, \mathrm{x}_{4}, \mathrm{x}_{5}\right\}$. The following steps are followed in this paper to design a residue to binary convertor based on MRC:

Step 1: Calculate the multiplicative inverse values,

Step 2: Substitute the calculated multiplicative inverse values in the MRC formulae and simplify the resultant equation by using arithmetic properties,

Step 3: Implement the simplified equations using hardware components such as full adders and logic gates.

In the next sections each step will be discussed in detail.

\subsection{Calculation of Multiplicative Inverse Values}

In this section, the required multiplicative inverse values, which are required in the MRC formula, will be examined. The calculations of the multiplicative inverse values are the most resource-consuming part of the reverse conversion. Thus, the goal of the moduli set design is to achieve a formula to simplify the computation of multiplicative inverses. Using the following propositions, we will simplify the reverse conversion formula.

Proposition 1. The multiplicative inverse of $\mathrm{m}_{1}=2^{\mathrm{n}}+3$ modulo $\mathrm{m}_{2}=2^{\mathrm{n}}+1$ is $2^{\mathrm{n}-1}+1$, in other words $\mathrm{I}\left(2^{\mathrm{n}}+\right.$ $3)\left.^{-1}\right|_{2^{n}+1}=2^{n-1}+1$.

Proof. $\left|\left(2^{\mathrm{n}-1}+1\right)\left(2^{\mathrm{n}}+3\right)\right|_{2^{\mathrm{n}}+1}=1$.

Proposition 2. The multiplicative inverse of $m_{1}=2^{n}+3$ modulo $\mathrm{m}_{4}=2^{\mathrm{n}}-1$ is $2^{\mathrm{n}-2}$, in other words $\mid\left(2^{\mathrm{n}}+\right.$ $3)\left.^{-1}\right|_{2^{\mathrm{n}}-1}=2^{\mathrm{n}-2}$

Proof. $\left|\left(2^{\mathrm{n}-2}\right)\left(2^{\mathrm{n}}+3\right)\right|_{2^{\mathrm{n}}-1}=1$.

Proposition 3. The multiplicative inverse of $\mathrm{m}_{2}=2^{\mathrm{n}}+1$ modulo $m_{3}=2^{n}$ is 1 , in other words $\left|\left(2^{n}+1\right)^{-1}\right|_{2^{n}}=1$.

Proof. $\left|\left(2^{\mathrm{n}}+1\right)\right|_{2^{\mathrm{n}}}=1$.

Proposition 4. The multiplicative inverse of $\mathrm{m}_{2}=2^{\mathrm{n}}+1$ modulo $\mathrm{m}_{4}=2^{\mathrm{n}}-1$ is $2^{\mathrm{n}-1}$, in other words $\mathrm{I}\left(2^{\mathrm{n}}+\right.$ 1) $\left.{ }^{-1}\right|_{2^{\mathrm{n}}-1}=2^{\mathrm{n}-1}$.

Proof. $\left|\left(2^{\mathrm{n}-1}\right)\left(2^{\mathrm{n}}+1\right)\right|_{2^{\mathrm{n}}-1}=1$.

Proposition 5. The multiplicative inverse of $m_{3}=2^{n}$ modulo $\mathrm{m}_{4}=2^{\mathrm{n}}-1$ is 1 , in other words $\left|\left(2^{\mathrm{n}}\right)^{-1}\right|_{2^{\mathrm{n}}-1}=1$.

Proof. $\left|\left(2^{\mathrm{n}}\right)\right|_{2^{\mathrm{n}}-1}=1$.

Proposition 6. The multiplicative inverse of $\mathrm{m}_{4}=2^{\mathrm{n}}-1$ modulo $m_{5}=2^{n}-3$ is $2^{n-1}-1$, in other words $\mid\left(2^{n}-\right.$ $1)\left.^{-1}\right|_{2^{\mathrm{n}}-3}=2^{\mathrm{n}-1}-1$.

Proof. $\left|\left(2^{\mathrm{n}-1}-1\right)\left(2^{\mathrm{n}}-1\right)\right|_{2^{\mathrm{n}}-3}=1$.

Other multiplicative inverse values, $A=\left|\left(2^{n}+3\right)^{-1}\right|_{2^{n}}$, $\mathrm{B}=\left|\left(2^{\mathrm{n}}+3\right)^{-1}\right|_{2^{\mathrm{n}}-3}, \quad \mathrm{C}=\left|\left(2^{\mathrm{n}}+1\right)^{-1}\right|_{2^{\mathrm{n}}-3}$ and $\mathrm{D}=$ $\left|\left(2^{\mathrm{n}}\right)^{-1}\right|_{2^{\mathrm{n}}-3}$ cannot either be formulated, or the formula is complicated. Therefore, we assume that these values are precalculated and saved using 4 in $n$ bits ROM. 
Table 1. Hardware and time requirements of the proposed converter

\begin{tabular}{cccccc} 
Components of MRC & $\mathbf{A}_{\mathbf{F A}}$ & $\mathbf{A}_{\mathbf{H A}}$ & $\mathbf{A}_{\text {AND }}$ & $\mathbf{A}_{\mathbf{N O T}}$ & $\boldsymbol{\tau}_{\mathrm{FA}}$ \\
\hline $\mathrm{d}_{2} \mathrm{~m}_{1}$ & $2 \mathrm{n}+2$ & $\mathrm{n}-1$ & & & $2 \mathrm{n}+2$ \\
$\mathrm{~d}_{3} \mathrm{~m}_{1} \mathrm{~m}_{2}$ & $\frac{1}{2} \mathrm{n}^{2}+\frac{3}{2} \mathrm{n}-1$ & & & $2 \mathrm{n}$ & $\mathrm{n}$ \\
$\mathrm{d}_{4} \mathrm{~m}_{1} \mathrm{~m}_{2} \mathrm{~m}_{3}$ & $3 \mathrm{n}+1$ & 1 & & $3 \mathrm{n}$ & $2 \mathrm{n}$ \\
$\mathrm{d}_{5} \mathrm{~m}_{1} \mathrm{~m}_{2} \mathrm{~m}_{3} \mathrm{~m}_{4}$ & $4 \mathrm{n}^{2}+11 \mathrm{n}+1$ & & $\mathrm{n}^{2}$ & & $5 \mathrm{n}$ \\
Total & $\sim 4 \mathrm{n}^{2}+11 \mathrm{n}$ & $\mathrm{n}$ & $\mathrm{n}^{2}$ & $5 \mathrm{n}$ & $5 \mathrm{n}$
\end{tabular}

\subsection{Simplifying MRC Formulae}

In this section, the multiplicative inverse values will be substituted in the MRC formulae in order to simplify the resultant equations. Based on the derived equations (1) and (2) in sub-section 2.2 and propositions given in sub-section 3.1 , the decoded value can be represented as $=\mathrm{d}_{1}+\mathrm{d}_{2} \mathrm{~m}_{1}+$ $\mathrm{d}_{3} \mathrm{~m}_{1} \mathrm{~m}_{2}+\mathrm{d}_{4} \mathrm{~m}_{1} \mathrm{~m}_{2} \mathrm{~m}_{3}+\mathrm{d}_{5} \mathrm{~m}_{1} \mathrm{~m}_{2} \mathrm{~m}_{3} \mathrm{~m}_{4}$, where the mixed radix digits can be obtained as:

$$
\begin{aligned}
& \mathrm{d}_{1}=\left|\mathrm{x}_{1}\right|_{\mathrm{m}_{1}}=\mathrm{x}_{1} \\
& \mathrm{~d}_{2}=\left.\left.\left|\left(\mathrm{x}_{2}-\mathrm{x}_{1}\right)\right| \mathrm{m}_{1}^{-1}\right|_{\mathrm{m}_{2}}\right|_{\mathrm{m}_{2}}=\left|\left(\mathrm{x}_{2}-\mathrm{x}_{1}\right)\left(2^{\mathrm{n}-1}+1\right)\right|_{2^{\mathrm{n}}+1} \\
& \mathrm{~d}_{3}=\left.\left.\left|\left(\left(\mathrm{x}_{3}-\mathrm{x}_{1}\right)\left|\mathrm{m}_{1}^{-1}\right|_{\mathrm{m}_{3}}-\mathrm{d}_{2}\right)\right| \mathrm{m}_{2}^{-1}\right|_{\mathrm{m}_{3}}\right|_{\mathrm{m}_{3}} \\
& =\left|\left(\mathrm{x}_{3}-\mathrm{x}_{1}\right) \mathrm{A}-\mathrm{d}_{2}\right|_{2^{\mathrm{n}}} \\
& \mathrm{d}_{4}=\left.\left.\left|\left(\left(\mathrm{x}_{4}-\mathrm{x}_{1}\right)\left|\mathrm{m}_{1}^{-1}\right|_{\mathrm{m}_{4}}-\mathrm{d}_{2}\left|\mathrm{~m}_{2}^{-1}\right|_{\mathrm{m}_{4}}-\mathrm{d}_{3}\right)\right| \mathrm{m}_{3}^{-1}\right|_{\mathrm{m}_{4}}\right|_{\mathrm{m}_{4}} \\
& =\left|\left(\left(\mathrm{x}_{4}-\mathrm{x}_{1}\right) 2^{\mathrm{n}-2}-\mathrm{d}_{2}\right) 2^{\mathrm{n}-1}-\mathrm{d}_{3}\right|_{2^{\mathrm{n}}-1} \\
& \mathrm{~d}_{5}=\mid\left(\left(\left(\left(\mathrm{x}_{5}-\mathrm{x}_{1}\right)\left|\mathrm{m}_{1}^{-1}\right|_{\mathrm{m}_{5}}-\mathrm{d}_{2}\right)\left|\mathrm{m}_{2}^{-1}\right|_{\mathrm{m}_{5}}-\mathrm{d}_{3}\right)\left|\mathrm{m}_{3}^{-1}\right|_{\mathrm{m}_{5}}\right) \\
& \left.\quad-\mathrm{d}_{4}\right)\left.\left|\mathrm{m}_{4}^{-1}\right|_{\mathrm{m}_{5}}\right|_{\mathrm{m}_{5}} \\
& =\left|\left(\left(\left(\left(\mathrm{x}_{5}-\mathrm{x}_{1}\right) \mathrm{B}-\mathrm{d}_{2}\right) \mathrm{C}-\mathrm{d}_{3}\right) \mathrm{D}-\mathrm{d}_{4}\right)\left(2^{\mathrm{n}-1}-1\right)\right|_{2^{\mathrm{n}}-3} .
\end{aligned}
$$

\subsection{Estimation of Implementation Cost}

In this section, the required hardware components to implement the simplified equations given in sub-section 3.2 will be calculated based on full adders and basic logic gates. The detailed hardware and time requirements of this converter are presented in Table 1 for each component. Note that $\boldsymbol{A}_{\mathrm{FA}}, \boldsymbol{A}_{\mathrm{HA}}, \boldsymbol{A}_{\mathrm{AND}}, \boldsymbol{A}_{\mathrm{NOT}}$ are the areas of a full-adder, halfadder, AND gate and inverter gate, respectively. Also, $\boldsymbol{\tau}_{\mathbf{F A}}$ is the delay of full-adder.

It is possible to reduce the conversion time at the expense of area. These use parallel adders to compute modulo adders/ modulo subtractors where based on the sign bits of the results, the correct value is selected. Parallel adders increase the area, but reduce the calculation time [11].

\section{PERFORMANCE COMPARISON OF REVERSE CONVERTERS}

In this Section the efficiency of the proposed RNS to binary converter is illustrated by comparing the hardware requirements. It is clear that the hardware requirements and conversion times depend on $n$ in the chosen moduli set. In Table 2, the total hardware requirements of the proposed converter are presented and compared to Cao [10], CICE and CIHS [11]. Furthermore, Table 2 shows that the proposed reverse converter has lower delay compared to other converters. Two later converters [11] are designed for four moduli set RNS. On the other hand the number of moduli increases the complexity of reverse converter significantly in terms of hardware requirements and conversion delay. Here, we choose Cao's design [10] to illustrate the efficiency of the proposed converter because it has almost the same operating range as the set that is proposed in this paper.

Table 2. Hardware and time complexity of the reverse converters

\begin{tabular}{ccccc} 
Design & $\boldsymbol{M}_{\boldsymbol{o p}}$ & Moduli Set & Area & Delay \\
\hline CICE [11] & $4 \mathrm{n}$ & $\left\{2^{\mathrm{n}}-3,2^{\mathrm{n}}-1,2^{\mathrm{n}}+1,2^{\mathrm{n}}+3\right\}$ & $\frac{5}{2} n^{2}+\frac{51}{2} n+12$ & $(18 \mathrm{n}+23) \tau_{\mathrm{FA}}$ \\
CIHS [11] & $4 \mathrm{n}$ & $\left\{2^{\mathrm{n}}-3,2^{\mathrm{n}}-1,2^{\mathrm{n}}+1,2^{\mathrm{n}}+3\right\}$ & $\frac{5}{2} n^{2}+\frac{75}{2} n+28$ & $(12 \mathrm{n}+15) \tau_{\mathrm{FA}}$ \\
Cao [10] & $5 \mathrm{n}$ & $\left\{2^{\mathrm{n}-1}-1,2^{\mathrm{n}}-1,2^{\mathrm{n}}, 2^{\mathrm{n}}+1,2^{\mathrm{n}+1}\right.$ & $\frac{5 \mathrm{n}^{2}+44 \mathrm{n}-4}{6}+16 \mathrm{n}-1$, & $>18 n \tau_{\mathrm{FA}}$ \\
& & $-1\}$ & $\mathrm{n}=6 \mathrm{k}-2$ & $>18 n \tau_{\mathrm{FA}}$ \\
Cao [10] & $5 \mathrm{n}$ & $\left\{2^{\mathrm{n}-1}-1,2^{\mathrm{n}}-1,2^{\mathrm{n}}, 2^{\mathrm{n}}+1,2^{\mathrm{n}+1}\right.$ & $\frac{5 \mathrm{n}^{2}+52 \mathrm{n}-12}{6}+16 \mathrm{n}-1$, & $\mathrm{n}=256 \mathrm{k}$ \\
Cao [10] & $5 \mathrm{n}$ & $\left\{2^{\mathrm{n}-1}-1,2^{\mathrm{n}}-1,2^{\mathrm{n}}, 2^{\mathrm{n}}+1,2^{\mathrm{n}+1}\right.$ & $\frac{5 \mathrm{n}^{2}+48 \mathrm{n}-8}{6}+16 \mathrm{n}-1$, & $>18 n \tau_{\mathrm{FA}}$ \\
& & $-1\}$ & $\frac{1}{2} \mathrm{n}^{2}+6 \mathrm{n}+a$ & $5 \mathrm{n} \tau_{\mathrm{FA}}$
\end{tabular}




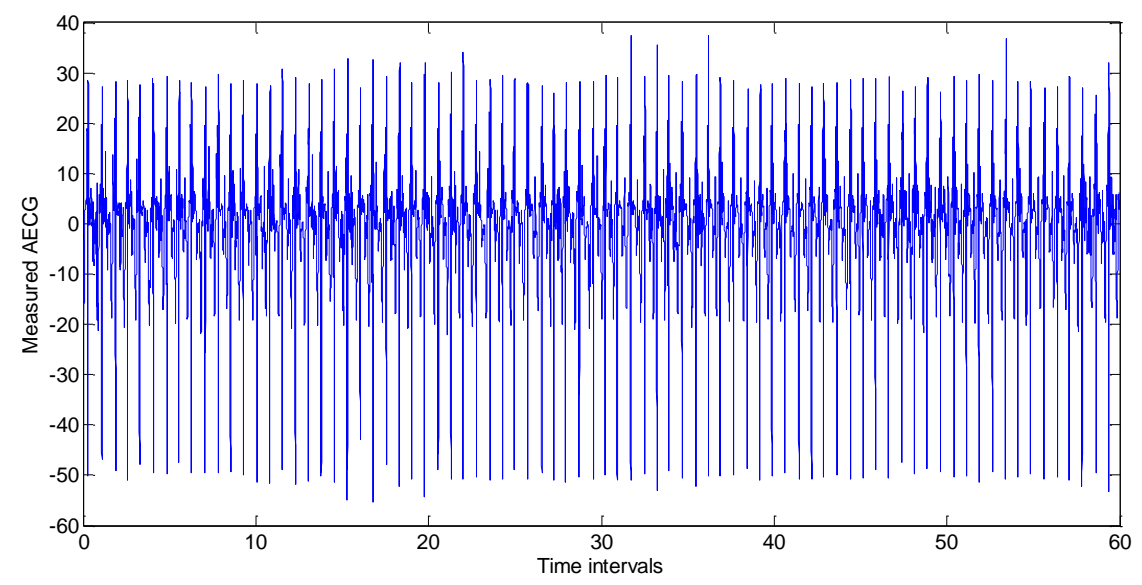

Fig 1: AECG signal from a01 PhysioBank's data collections

The main drawback of Cao's converter is that it is not designed for all values of $\mathrm{n}$ and it is restricted to $\mathrm{n}=6 \mathrm{k} \pm 2$. According to sub-section 3.1, the hardware requirements of $\mathrm{B}, \mathrm{C}, \mathrm{D}$ and $\alpha$ are estimated based on the area of full adder for different values of $\mathrm{n}$ and shown in Table 3 . The main aim of this paper is to develop an energy efficient design of reverse converter to be suitable for use in WBAN. Usually $n$ with smaller than 5 values are sufficient to cover the required operating range of $5 n$. Whereas, in [10], the smallest possible $\mathrm{n}$ values are 4 and after that 8 . This makes Cao's design unattractive for WBAN applications.

Table 3. Hardware requirements of $B, C, D$ and $\alpha$ based on $A_{F A}$ for different values of $n$

\begin{tabular}{ccccc}
$\mathbf{n}$ & $\mathbf{B}$ & $\mathbf{C}$ & $\mathbf{D}$ & $\boldsymbol{\alpha}$ \\
\hline 3 & 1 & 4 & 2 & 14 \\
4 & 11 & 10 & 9 & 49 \\
5 & 5 & 22 & 10 & 57 \\
6 & 51 & 46 & 41 & 123 \\
7 & 21 & 94 & 42 & 139
\end{tabular}

Figure 1 demonstrates the AECG (abdominal electrocardiogram) signal from a01 data collection which is extracted from PhysioBank [12]. The recorded AECG values are in the range $[-55.3,37.3]$. These values can easily be mapped to $[0,926]$ and can be represented by using 10 bits. Investigation of some other PhysioBank databases, such as mit100, showed that average number of 9 bits is sufficient for data representation. On the other hand by a small value of $n$ $=3$, the proposed five moduli set can cover the sensed physiological value with two redundant moduli. The main goal of this study is to apply the RNS as error detection and correction approach to improve the reliability of wireless data transmission. Thus, the existence of two redundant remainders means that the system is capable of detecting a burst error with maximum length of $2 n$ and correcting a burst error with maximum length of $n$.

Figure 2 compares the hardware requirements of proposed converter and Cao's design for different values of $n$ based on the number of required FAs. It can be seen that the hardware requirement of the proposed converter is $66 \%, 23 \%$ and $36 \%$ less than Cao's design for $n$ equals to three, four and five respectively.

\section{ANALYSIS AND DISCUSSION}

In this section, we will discuss the performance evaluation of the proposed reverse converter and the reliability of applying the studied five-moduli set on transmission errors in wireless communications.

The proposed moduli set, $\left\{2^{\mathrm{n}}+3,2^{\mathrm{n}}+1,2^{\mathrm{n}}, 2^{\mathrm{n}}-1,2^{\mathrm{n}}-3\right\}$, considered with the operating range of $3 n$ and two redundant moduli. Thus, applying this error control system enables the wireless transmission to detect a burst error with the length of up to $2 \mathrm{n}$ and correct a burst error with the length of up to $\mathrm{n}$. For example, when $n=3$ it is capable of transmitting 12 bit original data and handling 3 bits burst error. Whereas other known FEC methods add more redundant bits to cover the same number of burst errors in the same representation range. For instance, $\mathrm{BCH}(31,16)$ adds 15 redundant bits to each block of 16 bit original data to recover 3 bits burst error. The redundancy increases the transmission power consumption of sensor nodes significantly, since the radio unit is the most energy consuming-unit in sensor networks.

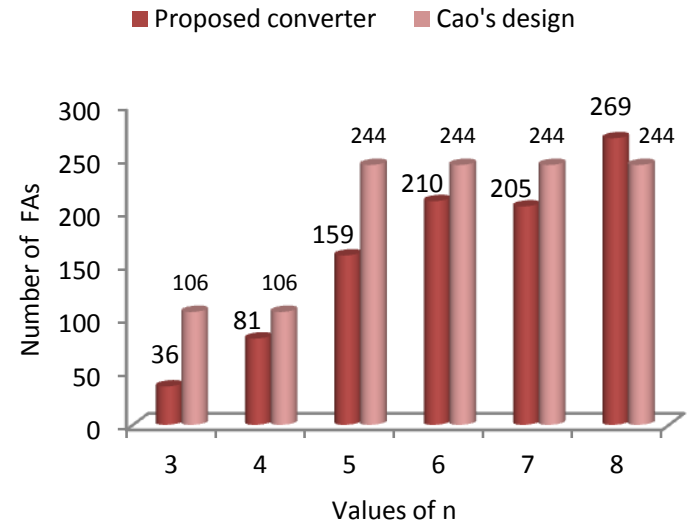

Fig 2: Hardware complexity: proposed converter vs Cao's 


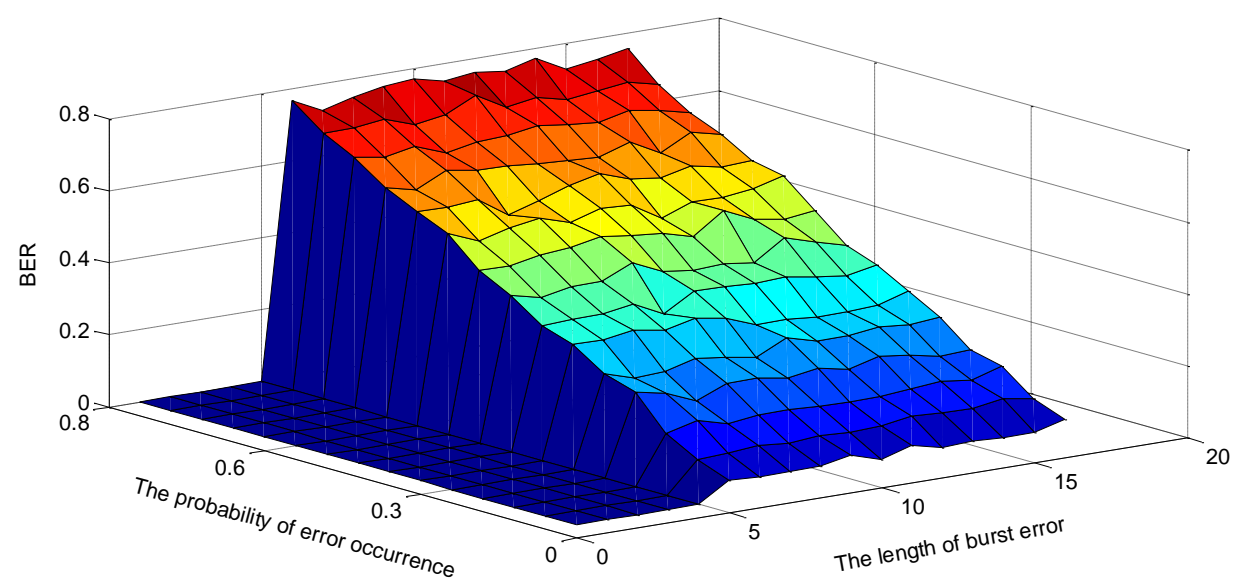

Fig 3. BER for different burst error length and probability

It has been shown in the previous section that the additional energy consumption for encoding binary values to RNS and decoding RNS to binary is reasonable by implementing the proposed reverse converter.

The Bit Error Rate of AECG signal can be seen in Figure 3. We injected a burst error into AECG signal to model the effect of wireless channel and to investigate the improvement of the proposed method with $n=5$. In [13], it was proven by experiments that long losses of information do happen with a non-negligible probability in the links between the nodes which is clearly not tolerable in a mission-critical WBAN setting. Thus, we implemented a function which injects the burst error to a block of data in MATLAB, to examine the reliability of the proposed system.

The 3D plot in Figure 3 shows that for the burst error lengths less that the remainder length of 5, the RNS code is capable of correcting all errors independent of the error occurrence probability. On the other hand, the proposed method cannot handle errors which have higher length than remainder length. In that case, the BER is constant depending on the probability of error occurrence. However, if the length of burst error is higher than the error correction capability of code, additional even number of redundant remainders could be considered as an option. This scenario requires another energy-efficient reverse converter which is not studied in this paper.

\section{CONCLUSION}

Commonly used FEC schemes incur significant communication overheads due to the inherent nature of the wireless sensors. An energy-efficient FEC has been proposed for WBAN. For the described reverse converter with fivemoduli set $\left\{2^{\mathrm{n}}+3,2^{\mathrm{n}}+1,2^{\mathrm{n}}, 2^{\mathrm{n}}-1,2^{\mathrm{n}}-3\right\}$ based on Mixed Radix Conversion method, the calculation and simplification of the decoder has been shown with reduced hardware and time complexity. Thus, the designed channel coding approach consumes less power compared to other recent RNS designs. Additionally, it is shown that in WBAN application, small values of $\mathrm{n}$ can cover the required operating range. For instance, $n=3$ is sufficient in a sensor network in which the sensor observes AECG. Thus, in the proposed moduli set, three of them are sufficient to cover the required operating range of physiological signals, and the other two can be considered as redundancy. Transmitting the physiological values using the proposed channel code enables the base station to handle burst errors up to the remainder length and increases the reliability of the wireless transmission. Detailed comparison of the proposed converter with other converters in the literature has been presented to demonstrate the trade-offs in combinational logic and conversion time.

\section{REFERENCES}

[1] M. A. Soderstrand, W. K. Jenkins, G. A. Jullien, and F. J. Taylor, Residue number system arithmetic: modern applications in digital signal processing: IEEE press, 1986.

[2] D. Gallaher, F. E. Petry, and P. Srinivasan, "The digit parallel method for fast RNS to weighted number system conversion for specific moduli $(2 \mathrm{k}-1,2 \mathrm{k}, 2 \mathrm{k}+1)$," Circuits and Systems II: Analog and Digital Signal Processing, IEEE Transactions on, vol. 44, pp. 53-57, 1997.

[3] Y. Wang, X. Song, M. Aboulhamid, and H. Shen, "Adder based residue to binary number converters for (2 n-1, 2 n, 2 n+ 1)," Signal Processing, IEEE Transactions on, vol. 50, pp. 1772-1779, 2002.

[4] F. Senel and M. Younis, "Optimized interconnection of disjoint wireless sensor network segments using $\mathrm{K}$ mobile data collectors," in ICC, 2012, pp. 492-496.

[5] A. Abbasi, M. Younis, and U. Baroudi, "Restoring connectivity in wireless sensor-actor networks with minimal topology changes," in Communications (ICC), 2010 IEEE International Conference on, 2010, pp. 1-5.

[6] B. Zarei, V. Muthukkumarasamy, and X.-W. Wu, "Residual Channel Coding in Low-Power WSNs Using Minimum Hamming Distance Decoder," presented at the International Conference on Advances in Computer and Electronics Technology - ACET 2014.

[7] B. Zarei, V. Muthukkumarasay, and X.-W. Wu, "A residual error control scheme in single-hop wireless sensor networks," in Advanced Information Networking and Applications (AINA), 2013 IEEE 27th International Conference on, 2013, pp. 197-204. 
[8] R. Conway and J. Nelson, "Improved RNS FIR filter architectures," Circuits and Systems II: Express Briefs, IEEE Transactions on, vol. 51, pp. 26-28, 2004.

[9] G. C. Cardarilli, A. Nannarelli, and M. Re, "Residue number system for low-power DSP applications," in Signals, Systems and Computers, 2007. ACSSC 2007. Conference Record of the Forty-First Asilomar Conference on, 2007, pp. 1412-1416.

[10] B. Cao, C.-H. Chang, and T. Srikanthan, "A residue-tobinary converter for a new five-moduli set," Circuits and Systems I: Regular Papers, IEEE Transactions on, vol. 54, pp. 1041-1049, 2007.

[11] P. V. A. Mohan, "New reverse converters for moduli set "International Journal of Electronics and Communications (AEU), vol. 29, pp. 643-658, 2008.
[12] Goldberger AL, Amaral LAN, Glass L, Hausdorff JM, Ivanov PCh, Mark RG, Mietus JE, Moody GB, Peng CK, Stanley HE. PhysioBank, PhysioToolkit, and PhysioNet: Components of a New Research Resource for Complex Physiologic Signals. Circulation 101(23):e215-e220 [Circulation Electronic Pages; http://circ.ahajournals.org/cgi/content/full/101/23/e215]; 2000 (June 13). PMID: 10851218.

[13] A. Natarajan, B. de Silva, K. Yap, M. Motani " Link Layer Behavior of Body Area Networks at $2.4 \mathrm{GHz}$ "MobiCom '09 Proceedings of the 15th annual international conference on Mobile computing and networking, ISBN: 978-1-60558-702-8. 